\title{
Skill training in Multimodal virtual environments
}

\author{
Daniel Gopher \\ Research Center for Work Safety and Human Engineering \\ Faculty of industrial engineering and Management \\ Technion, Haifa 32000 Israel
}

\begin{abstract}
Multimodal, immersive, virtual reality (VR) techniques open new perspectives for perceptual-motor skill trainers. They also introduce new risks and dangers. This paper describes the benefits and pitfalls of multimodal training and the cognitive building blocks of a multimodal, VR training simulators.
\end{abstract}

Keywords: Virtual reality, multimodal training, skill acquisition, perceptual motor skills, virtual reality training.

\section{Introduction}

Training simulators for complex tasks are being used in increased frequency since the end of the Second World War, which also marks the beginning of the technological age revolution. With the growing complexity of systems and their operation environments, the required duration of training and the increased costs of errors, on the job training became difficult or impossible and alternative simulation platforms have been developed to enable training. Flying, driving, space operation, surgery, power plant and process control are salient examples for tasks for which training simulators were developed. Early simulators were physical and mechanical analogues of their represented systems. With the advance of computer technology simulators become more and more hybrid and system dynamic, visual field and audition have been increasingly driven and generated by computers. Contemporary developments in sensors and display capabilities and the exponential increase in computation speed and storage capacity led the way to the development of multimodal virtual environments. In these environments, the operator is immersed, experience multimodal sensations and interacts with virtual objects including other humans [1]. Vision and audition have been in the study and design of simulators from their inception. The new and important addition is the inclusion of haptics: the ability to feel and exercise force, touch, texture and kinematics. Haptic technology is developing rapidly and haptic interfaces are now been incorporated in many virtual worlds. It is hence a quite conservative expectation that the multimodal, virtual reality platforms will dominate the next generation of training simulators.

At the outset of this discussion it is important to emphasize that topics of training and transfer of skills have not been in the focus of interest for the present research and development of virtual environments. When studying human behavior, task performance and interaction in virtual environments, the interest has been in the fundamentals of "virtualization" and the concepts of "presence" and "immersion". [2] propose the following definitions for the three concepts: Virtualization: "the process by which a human viewer interprets a patterned sensory impression to be an extended object in an environment other than that in which it physically exists" (p 332). "The interest is hence in the

\footnotetext{
${ }^{*}$ Corresponding author e-mail:
} 
properties and dimension that should be captured and represented in order to create virtual objects. Presence: "The common view is that presence is the sense of being in a VE rather than the place in which the participant's body is actually located" (P 333). Immersion: "...a person is immersed in an environment that is realized through computercontrolled display systems, and might be able to effect changes in that environment" (P 332).

When evaluating the value and possible contribution of VR technologies to the training of skills. Presence and Immersion are related but not the prime focus concern. The value of a training system is judged by relevance, its ability to provide relevant experience; by the provision of facilitation and guidance to the acquisition of the designated skill; by the transfer from VR training to performance in the real world. Relevance, facilitation and transferability are therefore the key constructs and the crucial criteria for the value of a training system, which are very different from presence and immersion. High level of presence and immersion may be important motivators and acquisition augmenters if there is a good match between the VR and real life experiences. However in case of mismatch or diversion, they may be harmful. High subjective sense of presence formed in spite of experiential diversions may lead to illusionary conjunctions, to reduced or even negative transfer. When developing training simulators, it is sometime important to create deliberate diversions and reduce physical fidelity to avoid involuntary and unconscious illusionary conjunctions.

\section{Training in multimodal environments}

Multimodal displays support flexible efficient communication, they are easy to learn, can be used in challenging situations, and people enjoy using them [3]. Multimodal interfaces succeed in creating a stronger sense of presence by better mimicking reality [4].The sensorial richness of multimodal environments translates into a more complete and coherent experience of the virtual world and therefore the sense of being present in the virtual realm is stronger(. An important advantage of the VR technology is the ease of providing augmented sensory feedback, visual guidance, auditory directors or augmented haptic cues.

\section{Developing training in multimodal environment}

To illustrate the application of these principles and concerns in the development of training platforms, we briefly describe platforms developed within the European Community $6^{\text {th }}$ framework project "Skills", "Multimodal Interfaces for Capturing and Transfer of Skills". The main building blocks of a training platform and a training program are:

1. A clear specification of the task to be learned, the skills to be acquired, the objectives of training, and the designated criteria of graduation.

2. Design of task scenarios, task versions and difficulty manipulation that best represent typical encounters and key requirements of the task. Richer and diverse training environment affords the development of a more flexible and higher level competence $[5,6]$.

3. Identification of key response and performance measures as well as progress criteria to evaluate trainee progress on relevant aspects of task performance and enhanced competence.

4. Definition of desired feedback indices and knowledge of results information to be given to trainees, as well as their frequency and mode of presentation.

When training is conducted as a preparatory stage, in a separate environment, or in a simulator, there is fifth important consideration:

5. Transfer of training. The relevance of the training experience in the learning environment determines the level of transfer from training to actual task performance.

Topics 2-4 have been instantiated through the development of accelerators and training protocols. The term accelerator is used to refer to variables that are introduced and implemented to facilitate, assist and improve learning. The term training protocol is employed to describe training schedule, duration, selected tasks scenarios, difficulty manipulations and their order of presentation. Six training platforms have been developed and evaluated: Rowing (ROW), Juggling (JUG), MaxilloFacial Surgery (MFS), Upper Limb Rehabilitation (ULR), Industrial Maintenance and Assembly (IMA), Programming by Demonstration (PBD) and. The IMA and PBD demonstrators include both Virtual Reality (VR) and Augmented Reality (AR) platforms; the ULR demonstrator includes Exoskeleton (Exos) and Bimanual (BM) systems. Table 1 presents a brief summary of the platform focus and their associated accelerators. 
Across the six platforms there are a total of 24 implemented accelerators. Ten of which, the dominant category, are feedback indicators.

Table 1

Six Multimodal VR training platforms and designed accelerators

\begin{tabular}{|c|c|c|c|}
\hline & Training Focus & $\begin{array}{l}\text { Targeted } \\
\text { population }\end{array}$ & Accelerators \\
\hline OW & $\begin{array}{l}\text { Acquisition of basic } \\
\text { rowing skills, effort and } \\
\text { energy manage-ment, } \\
\text { interpersonal coordination }\end{array}$ & $\begin{array}{l}\text { Novice and } \\
\text { Intermediate } \\
\text { rowers }\end{array}$ & $\begin{array}{l}\text { On line Visual spatial trajectory of rowing pattern } \\
\text { (Fd) } \\
\text { On line Vibration directive of rowing pattern (Fd) } \\
\text { Adjustable auditory pacer of the } \\
\text { locomotors/respiratory coupling (Rhythmic } \\
\text { Pacer) } \\
\text { Visual director of energy expenditure }(\mathbf{F d}) \\
\text { Visual and haptic information of interpersonal } \\
\text { coordination (Rhythmic Pacer) }\end{array}$ \\
\hline UG & $\begin{array}{l}\text { Attention management of } \\
\text { multiple moving objects, } \\
\text { spatial } \\
\text { temporal relationship, } \\
\text { bimanual rhythmic } \\
\text { coordination }\end{array}$ & $\begin{array}{l}\text { Novice } \\
\text { Jugglers }\end{array}$ & $\begin{array}{l}\text { Tactile-auditory rhythm trainer of juggling } \\
\text { coordination (Rhythmic Pacer) } \\
\text { Training at slow and gradually increasing task } \\
\text { speed (Task processing time) } \\
\text { Systematic exploration of the spatial temporal } \\
\text { components of the K dwell ratio (Control } \\
\text { strategy) }\end{array}$ \\
\hline FS & $\begin{array}{l}\text { Fine control of force } \\
\text { application, use of fine } \\
\text { graded touch and visual } \\
\text { information }\end{array}$ & $\begin{array}{l}\text { Trained } \\
\text { surgeons }\end{array}$ & $\begin{array}{l}\text { Feedback on forces and torques applied to the tool } \\
\text { (Fd) } \\
\text { Visual feedback on performance from an } \\
\text { "impossible" anatomical point of view }(\mathbf{F d}) \\
\text { Performance feedback relative to optimal } \\
\text { performance lines }(\mathbf{F d}) \\
\text { Multimodal feedback to enhance sensitivity to } \\
\text { compliance and vibration change }(\mathbf{F d})\end{array}$ \\
\hline MA & $\begin{array}{l}\text { Acquisition of procedural } \\
\text { skills in virtual } \\
\text { environment and via a } \\
\text { remote augmented reality } \\
\text { training }\end{array}$ & $\begin{array}{l}\text { Technicians } \\
\text { and machine } \\
\text { operators }\end{array}$ & $\begin{array}{l}\text { Including haptic in 3D VR training (Hp Enact) } \\
\text { Adding abstract representation to enaction (Cog. } \\
\text { Aid) } \\
\text { Introducing direct visual aid (pointer) (Vis. } \\
\text { Director) } \\
\text { Adding images of parts (Cog. Aid) } \\
\text { Adding rotational haptic hints (Hp Enaction) } \\
\text { Augmenting enaction by theoretical instructions } \\
\text { (Cog. Aid) }\end{array}$ \\
\hline $\mathrm{BD}$ & $\begin{array}{l}\text { Exploring and adapting } \\
\text { behavior to the motion } \\
\text { and compliance } \\
\text { constraints of a robotic } \\
\text { arm }\end{array}$ & $\begin{array}{l}\text { PBD robot } \\
\text { operators }\end{array}$ & $\begin{array}{l}\text { On line indicators of approaching singularity (Fd) } \\
\text { Voluntary exploration of singularity (Control } \\
\text { strategy). } \\
\text { Haptic exploration of compliance parameters } \\
\text { setting (Hp. Enaction) }\end{array}$ \\
\hline LR & $\begin{array}{l}\text { Using robotic technology } \\
\text { and VR to expand } \\
\text { rehabilitation options / } \\
\text { interaction }\end{array}$ & $\begin{array}{l}\text { Patients } \\
\text { undergoing } \\
\text { limb control } \\
\text { physiother. }\end{array}$ & $\begin{array}{l}\text { Task selection } \\
\text { On line continuous feedback }(\mathbf{F d}) \\
\text { Motion adaptation (Fd, Motivation) }\end{array}$ \\
\hline
\end{tabular}

Accelerators capitalize on the elaborated measurement and capturing techniques incorporated in each of the demonstrators, to provide trainees with experiential, on line feedback on their performance which could have not been presented otherwise. In most cases, the information provided to the learner is enacted by the learner him/herself (e.g. energy consumption in rowing), and feedback relate to the discrepancy between current and target values. Feedback indicators vary in their modality of presentation (visual, tactile, auditory), their time mode (continuous versus intermittent) and reference 
point (Trainee performance level, hitting boundaries or constraints, correspondence to expert or optimal performance models). There are three important tests for the value of feedback based accelerators are: a) The relevance of the information and type of guidance to learning; b) the ability to improve learning without developing dependence on the feedback presence, which will degrade performance immediately once this feedback is removed; c) the presence of feedback should not distract or interfere with the regular modes of performing the trained task. These aspects have been examined in the platforms evaluation studies.

Assessing the value of the developed platforms for skill acquisition and the best ways of applying them in training is a multifaceted task. There are four basic evaluation aspects that need to be examined: 1) A comparative evaluation of the differential experience of performing the same tasks on the VR platform and in the real world; 2) Evaluation of the contribution of accelerators; 3) Assessment of training protocols that will maximize learning and skill acquisition on a platform; 4) Transfer of training studies. The first type of evaluation aims to identify the similarities and differences between performing the same tasks (e.g. rowing, juggling, drilling) in the real world and in the VR training platform. Such an assessment is crucial to better understand the differences between acquiring a skill in the virtual and in the real environment, and the possible implications of these differences on the use of the VR platform in training and transfer. The main question is whether expert performers can comfortably employ the same form of behavior and execute their acquired skills, in the virtual as in the real environment. This question extends much beyond the subjective feeling of immersion or presence. In summary from many perspective it is clearly shown that from a human performance as well as skill acquisition perspective, the new multimodal VR technologies offer new and exciting potential for the development of simulators and the training of complex skills [7]. At the same time, they present nontrivial challenges that should be carefully evaluated and avoided.

\section{References}

[1] G. Riva, Virtual Reality.Wiley Encyclopedia of Biomedical Engineering, John Wiley \& Sons, Inc. (2006).

[2] M.V. Sanchez-Vives, M. Slater, From presence to consciousness through Virtual Reality. Nature Reviews, 6, (2005), pp 332-339

[3] S. Oviatt, Multimodal Interfaces. In J. Jacko \& A. Sears (Eds.): Handbook of Human-Computer Interaction (2002) Mahwah: New Jersey: Lawrence Erlbaum, (pp. 286-304).

[4] D.M. Romano, \& P. Brna, Presence and reflection in training: Support for learning to improve quality decisionmaking skills under time limitations. Cyber Psychology and Behavior, 4(2), (2001), 265-278.

[5] R. Schmidt \& R. Bjork, "New Conceptualization of Practice: Common Principles in Three Paradigms Suggest New Concepts for Training”. Psychological Science, (1992), pp 207-217.

[6] D. Gopher, D. Emphasis change a training protocol for high demands tasks. In: A. Kramer, D. Wiegman. A. Kirlik (Eds): Applied Attention: From Theory to Practice. (2007), Oxford Psychology Press, pp 207-224.

[7] J. M. Weller, Simulation in undergraduate medical education: bridging the gap between theory and practice. Medical Education, 38, (2004), pp 32-38. 